\title{
O ensino da Saúde do Trabalhador nos cursos de graduação em saúde de uma universidade federal
}

\author{
The teaching of Health of Worker in the undergraduate health courses of a federal university \\ La enseñanza de la Salud de los Trabajadores en los cursos de salud de pregrado en una \\ universidad federal
}

Recebido: 19/09/2021 | Revisado: 29/09/2021 | Aceito: 30/09/2021 | Publicado: 02/10/2021

\author{
Diego de Oliveira Souza \\ ORCID: https://orcid.org/0000-0002-1103-5474 \\ Universidade Federal de Alagoas, Brasil \\ E-mail: diego.souza@ arapiraca.ufal.br
}

\begin{abstract}
Resumo
Apesar de ser um campo relativamente novo, a Saúde do Trabalhador suscitou debates importantes para a Reforma Sanitária brasileira, o que implicou novas possibilidades e desafios para a formação de profissionais de saúde no Brasil. Tendo esse contexto em vista, esse estudo buscou analisar o ensino da Saúde do Trabalhador nos cursos de graduação em saúde da Universidade Federal de Alagoas (Ufal). Trata-se de um estudo qualitativo, descritivo, de base documental. Foram analisados os Projetos Pedagógicos dos Cursos de saúde da Ufal. Contatou-se que três desses cursos possuem disciplinas ou módulos relacionados à Saúde do Trabalhador. A pouca amplitude que o campo em questão possui no ensino em saúde pode estar associada à sua pouca difusão pela rede de saúde, figurando como atenção especializada, quando deveria ser transversal ao sistema.
\end{abstract}

Palavras-chave: Ensino; Educação profissional em saúde pública; Saúde do trabalhador.

\begin{abstract}
Health of Worker raised important debates for the Brazilian Sanitary Reform, which implied new possibilities and challenges for the training of health professionals in Brazil, despite being a relatively new field. With this context in mind, this study sought to analyze the teaching of Health of Worker in undergraduate health courses at the Universidade Federal de Alagoas (Ufal). This is a qualitative, descriptive, documentary-based study. The Pedagogical Projects of the health courses at Ufal were analyzed. It was found that three of these courses have disciplines or modules related to Health of Worker. The little amplitude that the field in question has in health teaching may be associated with its little diffusion by the health network, figuring as specialized care, when it should be transversal to the system.
\end{abstract}

Keywords: Teaching; Education, public health professional; Health of worker.

\section{Resumen}

A pesar de ser un campo relativamente nuevo, la Salud del Trabajador planteó importantes debates para la Reforma Sanitaria brasileña, lo que implicó nuevas posibilidades y desafíos para la formación de los profesionales de la salud en Brasil. Teniendo en cuenta este contexto, este estudio buscó analizar la enseñanza de la Salud del Trabajador en los cursos de pregrado en salud de la Universidad Federal de Alagoas (Ufal). Se trata de un estudio cualitativo, descriptivo y documental. Se analizaron los Proyectos Pedagógicos de los cursos de salud de la Ufal. Se comprobó que tres de estos cursos tienen disciplinas o módulos relacionados con la salud de los trabajadores. La poca amplitud que el campo en cuestión tiene en la enseñanza de la salud puede estar asociada a su poca difusión por la red sanitaria, figurando como atención especializada, cuando debería ser transversal al sistema.

Palabras clave: Enseñanza; Educación en salud pública profesional; Salud del trabajador.

\section{Introdução}

A Saúde do Trabalhador é um campo científico e institucional relativamente recente, cujas bases emergiram das lutas operárias realizadas em meados do século XX, notadamente o movimento operário italiano (décadas de 1960 e 1970) e as experiências latino-americanas (décadas de 1970 e 1980). Em contraposição a outros modelos de investigação e intervenção sobre a relação trabalho-saúde (a exemplo da Medicina do Trabalho e da Saúde Ocupacional), a Saúde do Trabalhador nasce 
da experiência dos próprios trabalhadores, sob aliança com técnicos da saúde, preocupada com a transformação dos processos de trabalho (Mendes \& Dias, 1994; Vasconcellos, 2011; Souza, 2019).

No caso brasileiro, a Saúde do Trabalhador se constituiu, também, como campo de militância na década de 1980, exercendo alguma influência nos primeiros passos dados pelo Movimento da Reforma Sanitária, do qual se originou o Sistema Único de Saúde (SUS), ao final da década de 1980. Reverberou academicamente, constituindo-se como disciplina ou área de especialização em diversas áreas da saúde, a reboque do processo de institucionalização do SUS, na década de 1990 (Souza, 2019). Esperava-se que o SUS, enquanto sistema pautado em uma concepção de saúde como processo social fundado pelo trabalho, daria a mesma ênfase à categoria trabalho no âmbito institucional, nos seus programas e serviços. No entanto, a Saúde do Trabalhador (enquanto campo que confere tal centralidade ao trabalho) nunca assumiu eminência no SUS, ficando em uma posição periférica, sem muita aderência à totalidade da rede de saúde (Leão \& Vasconcellos, 2011).

Diante disso, podemos afirmar que se constitui um novo campo, capaz de criar tensões no status quo e abrir novas perspectivas e demandas na formação dos profissionais de saúde ${ }^{1}$. Porém, esse campo se mistura às velhas maneiras de enxergar a saúde no trabalho, obstando-lhe o potencial crítico e retroalimentando sua posição periférica no SUS, o que pode ser um reflexo, entre outras coisas, da inconsistência da formação no que tange a essa área.

Com efeito, entender o que se ensina sobre a Saúde do Trabalhador nos cursos de graduação em saúde é um passo importante para efetivar as novas possibilidades de atuação profissional e de transformação em nível institucional, no sentido de conferir maior visibilidade ao campo em questão. Para tanto, alguns estudos sobre o ensino da Saúde do Trabalhador têm sido realizados no Brasil, apontando seu grau de inserção nos variados cursos, aspectos mais abordados, obstáculos e avanços, com destaque para a maior presença de pesquisas sobre a graduação em Enfermagem.

Monteiro et al. (2007) relataram a experiência do ensino sobre Vigilância em Saúde do Trabalhador (VISAT) na disciplina de Enfermagem em Saúde do Trabalhador em uma universidade federal. Os autores avaliam que o conhecimento dessa área é imprescindível para a Enfermagem, sobretudo diante do envelhecimento dos trabalhadores e exposição aos riscos do processo de trabalho por mais tempo. Com isso, o relato destaca a centralidade da VISAT para a área, o que implica estratégias de ensino que aproximem os estudantes aos dados da realidade a ser vigiada. Pensando nisso, a disciplina em questão possibilitou a análise descritiva de 852 Comunicações de Acidente de Trabalho (CAT), gerando bons resultados de aprendizagem e sendo avaliada positivamente pelos alunos.

Em pesquisa qualitativa com discentes de um curso de Enfermagem, Marques et al. (2012) constataram que, quando se trata de Saúde do Trabalhador, os seguintes conteúdos são abordados: atuação do enfermeiro em Saúde do Trabalhador, riscos ocupacionais e classificação de risco, Equipamentos de Proteção Individual (EPI), CAT, prevenção de acidentes, leis e direito trabalhista, processo de trabalho e nexo com a saúde-doença do trabalhador. Contudo, alertam que a relação teoria/prática é incipiente e fragilizada, o que dificulta a visualização desses conteúdos na realidade.

Fernandes, Souza e Marques (2013) fizeram um levantamento bibliográfico sobre a produção científica a respeito da mesma questão, também em cursos de Enfermagem. Constataram que os cursos de graduação abordam diversos aspectos dessa área, a exemplo da VISAT, acidentes de trabalho, investigação de acidentes de trabalho, epidemiologia aplicada à Saúde do Trabalhador, riscos e doenças ocupacionais, Saúde do Trabalhador no SUS, entre outros. Concluíram que existe:

\footnotetext{
${ }^{1} \mathrm{O}$ ensino de disciplinas sobre a relação trabalho-saúde ou, até mesmo, cursos de Medicina do Trabalho, Higiene Industrial ou Engenharia de Segurança se faziam presentes, no Brasil, desde os anos 1950 e, com maior ênfase, na década de 1970. Contudo, o que estamos considerando como Saúde do Trabalhador consiste em um novo campo, distinto dessas abordagens pregressas, ainda que com alguns elementos comuns (voltaremos a essas diferenças por ocasião da discussão dos resultados). Portanto, apenas se pode falar em ensino da Saúde do Trabalhador nas universidades, após a construção dessa nova área, no final da década de 1970 e início da década de 1980.
} 
[...] a relevância de os cursos de graduação de enfermagem adotarem o referencial teórico sobre saúde do trabalhador; inserindo e/ou aprimorando os conteúdos sobre saúde e trabalho com a perspectiva de sensibilização dos estudantes acerca do processo de adoecimento em decorrência do trabalho (Fernandes; Souza \& Marques, 2013, p. 259).

Em outro estudo, Fernandes et al. (2013) destacam algumas limitações para o ensino da Saúde do Trabalhador, como o pouco conhecimento dos docentes sobre o conteúdo e o pouco interesse/procura dos discentes por atividades da área. Além disso, informam que há discrepâncias entre o currículo formal (quando consta a Saúde do Trabalhador como conteúdo) e o currículo efetivado na prática, muitas vezes não abordando ou apenas abordando superficialmente a temática.

Além desses estudos sobre a Saúde do Trabalhador nos cursos de Enfermagem, encontramos estudos que discutem o tema nos cursos de Medicina, Odontologia e Terapia Ocupacional. No caso da Medicina, a investigação se deu em um curso com tradição na abordagem das relações trabalho-saúde-doença em seu currículo. Dias et al. (2006) buscaram avaliar o programa da disciplina Saúde do Trabalhador, a partir da percepção dos estudantes. Os principais achados do estudo giram em torno de três questões: o ensino da anamnese ocupacional parece não surtir muito efeito, pois a maioria dos estudantes não a realiza quando a relação da doença com o trabalho não está explícita; já em relação aos riscos ocupacionais, a eficácia tem sido maior, pois os estudantes passaram a investigá-los quando deparam com indivíduos com doenças relacionadas ao trabalho; e o resultado também tem sido positivo para os encaminhamentos trabalhistas e previdenciários dos trabalhadores. Os autores apontam a dificuldade em proporcionar atendimento dos estudantes aos trabalhadores durante a disciplina em si, devido às questões estruturais da universidade, com turmas de 160 alunos e ambulatórios fisicamente pequenos.

Em trabalho de revisão, Perin et al. (2012) destacam que a Saúde do Trabalhador é um novo conteúdo para a Odontologia. O interesse pela área vem crescendo desde que, em 2001, o Conselho Federal de Odontologia instituiu a Odontologia do Trabalho como nova especialização; porém, a graduação ainda está alheia aos conteúdos dessa nova área. Os autores defendem que essa aproximação deve ocorrer desde a graduação, pois a necessidade de programas de saúde bucal para os trabalhadores vem aumentando rapidamente, o que demanda uma resposta na formação profissional em Odontologia.

Já a Terapia Ocupacional pode ser considerada uma profissão mais jovem do que as já citadas Enfermagem, Medicina e Odontologia, uma vez que passou a ser regulamentada como profissão, no Brasil, em 1969 e, mais expressivamente, ampliou sua atuação na década de 1980. Porém, pela sua dinâmica própria, com o uso terapêutico das ocupações, possui uma relação mais substantiva com as disciplinas científicas afins ao mundo do trabalho. Isso tem possibilitado uma inserção mais consistente da Saúde do Trabalhador nessa profissão do que em outras, mais tradicionais, a exemplo da Odontologia. No estudo qualitativo de Pereira Jorge et al. (2015), com estudantes de Terapia Ocupacional de 15 universidades públicas, constatou-se a presença de conteúdos de Saúde do Trabalhador em todos os cursos, com muitos professores sendo especialistas em áreas afins, como a Ergonomia. A inserção se mostra ainda mais consistente, pois esses cursos possuem projetos de extensão e pesquisa voltados à Saúde do Trabalhador, com professores e discentes produzindo e publicando textos em periódicos sobre o assunto.

Diante disso, observamos avanços e desafios no âmbito do ensino da Saúde do Trabalhador, o que nos levou ao seguinte questionamento: como se organiza o ensino da Saúde do Trabalhador na Universidade Federal de Alagoas (Ufal)? Para contribuir com a resolução desse questionamento, apresentamos essa pesquisa, com o objetivo de analisar o ensino da Saúde do Trabalhador nos cursos de graduação em saúde da Ufal a maior e mais antiga instituição de ensino superior do estado. 


\section{Metodologia}

Metodologicamente, estruturamos um estudo qualitativo, descritivo, do tipo documental. O escopo de documentos foi constituído pelos Projetos Pedagógicos dos Cursos (PPC)² das graduações em saúde da Ufal. Há três campi dessa universidade, mas no campus Sertão não existe curso da área da saúde. Nos outros dois campi - campus Aristóteles Calazans Simões (A. C. Simões) em Maceió e campus Arapiraca - há sete cursos de saúde, conforme demonstramos no Quadro 1.

Quadro 1. Cursos de saúde da Ufal de acordo com campus e ano do PPC analisado.

\begin{tabular}{|l|l|l|}
\hline Curso & Campus & Ano de aprovação do PPC \\
\hline Enfermagem & Campus A. C. Simões & 2007 \\
\hline Enfermagem & Campus Arapiraca & 2018 \\
\hline Farmácia & Campus A. C. Simões & 2007 \\
\hline Medicina & Campus A. C. Simões & 2013 \\
\hline Medicina & Campus Arapiraca & 2018 \\
\hline Nutrição & Campus A. C. Simões & 2010 \\
\hline Odontologia & Campus A. C. Simões & 2007 \\
\hline
\end{tabular}

Fonte: Autores.

Os PPC foram lidos na íntegra, com foco para as disciplinas ou módulos que compõem as matrizes curriculares. Buscamos a presença de disciplinas/módulos específicos sobre a Saúde do Trabalhador ou campos correlatos à relação trabalho-saúde. Ainda verificamos as ementas e a bibliografia de disciplinas ou módulos mais amplos que demonstrem potencial para abordar a Saúde do Trabalhador como tópico pontual ou indiretamente. Agrupamos os resultados dos sete cursos de acordo com suas semelhanças, discutidos criticamente a seguir.

\section{Resultados e Discussão}

Inicialmente, destacamos que os cursos de Farmácia, Enfermagem do campus A. C. Simões, Nutrição e Odontologia não possuem componente curricular específico para a Saúde do Trabalhador. A temática também não comparece explicitamente na ementa de outras disciplinas/módulos, nem com referências sobre a temática. Já os cursos de Enfermagem do campus Arapiraca e os dois cursos de Medicina possuem componentes curriculares que abordam diretamente a Saúde do Trabalhador, estruturados sob a forma de módulos, conforme Quadro 2.

\footnotetext{
${ }^{2}$ Universidade Federal de Alagoas (2007a; 2007b; 2007c; 2010; 2013; 2018a; 2018b).
} 
Quadro 2: Disciplinas/módulos obrigatórios sobre Saúde do Trabalhador nos cursos de Saúde da Ufal.

\begin{tabular}{|c|c|c|c|}
\hline $\begin{array}{l}\text { Componente } \\
\text { Curricular }\end{array}$ & Curso & $\begin{array}{l}\text { Carga } \\
\text { horária }\end{array}$ & Ementa \\
\hline $\begin{array}{l}\text { Gerenciamento/assistência de } \\
\text { Enfermagem à Saúde do } \\
\text { Trabalhador }\end{array}$ & $\begin{array}{l}\text { Enfermagem - } \\
\text { Arapiraca }\end{array}$ & 18 & $\begin{array}{l}\text { Estuda o processo saúde-doença em sua relação com o } \\
\text { trabalho, as respostas sociais dadas a esse processo, } \\
\text { sobretudo no âmbito das políticas públicas de saúde. } \\
\text { Destaca o trabalho em Enfermagem voltado à saúde do } \\
\text { trabalhador, levando em consideração, em especial, as } \\
\text { demandas institucionais engendradas no Sistema Único de } \\
\text { Saúde (Universidade Federal de Alagoas, 2018a). }\end{array}$ \\
\hline $\begin{array}{l}\text { Biossegurança e Saúde do } \\
\text { Trabalhador(eletiva) }\end{array}$ & $\begin{array}{l}\text { Enfermagem - } \\
\text { Arapiraca }\end{array}$ & 18 & Ausente \\
\hline $\begin{array}{l}\text { Integração Ensino-Comunidade } \\
\text { e Serviço } 4\end{array}$ & $\begin{array}{l}\text { Medicina } \\
\text { Arapiraca }\end{array}$ & 72 & $\begin{array}{l}\text { Vigilância em saúde. Vigilância sanitária, vigilância } \\
\text { ambiental, vigilância epidemiológica, de saúde do } \\
\text { trabalhador e hospitalar (Universidade Federal de } \\
\text { Alagoas, 2018b, grifo nosso) }\end{array}$ \\
\hline $\begin{array}{l}\text { Integração Ensino-Comunidade } \\
\text { e Serviço } 7\end{array}$ & $\begin{array}{l}\text { Medicina } \\
\text { Arapiraca }\end{array}$ & 72 & $\begin{array}{l}\text { Estudo dos problemas de saúde provocados ou agravados } \\
\text { pelo trabalho. Avaliação dos riscos ocupacionais. } \\
\text { Apresentação dos procedimentos e ferramentas para } \\
\text { investigação dos agravos à saúde relacionados com o } \\
\text { trabalho, no nível individual e coletivo. Análise do quadro } \\
\text { de saúde dos trabalhadores no Brasil, em seus aspectos } \\
\text { clínico-epidemiológicos e das condutas médicas e } \\
\text { previdenciárias frente às causas de morbidade mais } \\
\text { prevalentes. Organização da atenção à saúde dos } \\
\text { trabalhadores: atuação do Estado, dos empregadores e } \\
\text { trabalhadores. Política Nacional de Saúde do Trabalhador e } \\
\text { da Trabalhadora (Universidade Federal de Alagoas, } \\
\text { 2018b). }\end{array}$ \\
\hline Saúde e Sociedade VII & $\begin{array}{l}\text { Medicina - A. } \\
\text { C. Simões }\end{array}$ & 34 & $\begin{array}{l}\text { Introdução do discente nos conceitos básicos da medicina } \\
\text { do trabalho. Habilitar nas técnicas propedêuticas e } \\
\text { habilidades de diagnóstico clínico, laboratorial, tratamento } \\
\text { e prevenção das principais patologias do trabalho } \\
\text { (Universidade Federal de Alagoas, 2013). }\end{array}$ \\
\hline
\end{tabular}

Fonte: Autores, a partir dos PPC.

Convém destacar que Enfermagem e Medicina, de fato, são os cursos com maior inserção e tradição na Saúde do Trabalhador ou em campos congêneres, a exemplo da Saúde Ocupacional. Os profissionais dessa área são listados como integrantes obrigatórios de equipes de Serviços Especializados em Engenharia de Segurança e em Medicina do Trabalho nas empresas privadas ou nos Centros de Referência em Saúde do Trabalhador - Cerest's, no âmbito do SUS. Essa condição reflete a presença de tópicos desse campo no ensino de graduação, a exemplo do que outros autores já tinham constatado (Dias et al., 2006; Monteiro et al., 2007; Fernandes et al., 2013; Fernandes; Souza \& Marques, 2013).

Um aspecto importante a ser observado é que um dos módulos da Medicina do campus Arapiraca listado no Quadro 2 não consiste em uma abordagem exclusiva da Saúde do Trabalhador, mas aborda a Visat no contexto mais amplo da Vigilância em Saúde. Todavia, é pertinente destacar esse módulo no referido Quadro, por tratar do elemento que, para alguns autores (Monteiro et al., 2007; Vasconcellos, 2011), é estruturante do campo em questão.

Salientamos que o curso de Medicina do campus A. C. Simões parece estar mais restrito à Medicina do Trabalho, enquanto os outros dois estão mais próximos, decerto, da Saúde do Trabalhador, implicando diferenças importantes. Isso porque a Medicina do Trabalho se distingue da Saúde do Trabalhador desde a sua origem, face à Revolução Industrial europeia (século XIX), quando nasce ligada às necessidades do patronato em conter a influência negativa que o adoecimento dos trabalhadores provocava na produtividade, assim como marcada pela ideia de objetificação do trabalhador, em um modelo 
centrado da autoridade médica e na patologia do trabalho (esses últimos aspectos presentes com destaque na ementa do módulo Saúde e Sociedade VII - Medicina do campus A. C. Simões).

Os cursos do campus Arapiraca não estão isentos dessa perspectiva mais conservadora, até porque ela reflete o mix existente na rede de saúde (Souza, 2019). Exemplo disso é a perspectiva de biossegurança presente no módulo eletivo do curso de Enfermagem do campus Arapiraca, ainda que nele se amplie o "olhar" para além da figura do médico. Apesar disso, a biossegurança ainda está inserida numa perspectiva que separa saúde e segurança para depois, gnosiologicamente, uni-las (em uma prática de justaposição típica da Saúde Ocupacional), ao passo que Saúde do Trabalhador parte da ideia de totalidade, onde segurança está contida na saúde e, seja qual for a hipótese ou situação, o sujeito do processo deve ser o próprio trabalhador (Vasconcellos, 2011; Minayo-Gomez \& Thedim-Costa, 1997; Souza, 2019). Portanto, campos como Saúde Ocupacional, Engenharia de Segurança e Biossegurança se aproximam muito mais da Medicina do Trabalho do que da Saúde do Trabalhador.

Não obstante, a diferença entre Medicina do Trabalho, Saúde Ocupacional e Saúde do Trabalhador não é meramente semântica, mas expressa um instrumental teórico-metodológico distinto e, sobretudo, uma posição diferente da classe trabalhadora no processo. Enquanto os dois primeiros campos estão preocupados com a reprodução da força de trabalho, a Saúde do Trabalhador está preocupada em alçar o trabalhador ao seu papel de agente transformador do processo de trabalho, naquilo que tange a saúde. Por isso, encontra um terreno mais fecundo para se desenvolver no âmbito da Saúde Pública, a exemplo do SUS no Brasil, aspecto bastante enfatizado nas ementas dos cursos de Enfermagem e Medicina do campus Arapiraca.

Quando observadas as disciplinas ou módulos que não manifestam, explicitamente, a abordagem da relação trabalhosaúde, mas que demonstram um potencial temático para tal, podemos incluir os sete cursos. Em todos eles existem disciplinas ou módulos que abordam a aplicação das Ciências Humanas e Sociais na saúde, Saúde Coletiva/Saúde Pública, Epidemiologia ou Vigilância em Saúde. Por ocasião dessas disciplinas ou conteúdos, ainda que não seja citada a Saúde do Trabalhador diretamente, ela pode comparecer de diferentes formas: como fenômeno social oriundo das relações sociais de produção (perspectiva das Ciências Humanas e Sociais); como serviço estruturado na rede pública (a exemplo dos Cerest) nas disciplinas de Saúde Coletiva/Saúde Pública; ou como dado epidemiológico ou objeto de Vigilância em Saúde, nas disciplinas ligadas à Epidemiologia.

Essa correlação indireta é válida, porquanto a Saúde do Trabalhador está inserida em um campo mais amplo, designado Saúde Coletiva, embora, nem sempre, com a ênfase que deveria ter por dentro dele (Minayo-Gomez \& ThedimCosta, 1997). Considerando a complexidade da Saúde Coletiva, composta por eixos que envolvem as Ciências Sociais e Humanas, a Epidemiologia e a Gestão, componentes curriculares que envolvam qualquer desses eixos, potencialmente, podem ser espaços para o debate da Saúde do Trabalhador.

Do ponto de vista metodológico, a análise mais genérica dos PPC não possibilita a apreensão de particularidades pedagógicas em cada componente curricular. Contudo, cada PPC expressa as tendências pedagógicas (que se desdobram em estratégias metodológicas) predominantes no curso, de modo genérico. Por exemplo, é possível identificar se o curso se baseia em metodologias tradicionais ou ativas.

Diante dessa condição e considerando que a Saúde do Trabalhador exige uma pedagogia contra hegemônica, com papel ativo dos trabalhadores (Vasconcellos; Almeida \& Guedes, 2009), aqueles cursos que escapam das abordagens tradicionais, com maior inserção de metodologias ativas, possuem potencial de maior aproximação dos discentes com a realidade da classe trabalhadora. Assim, esses cursos também possuem maiores chances de desenvolver processos de aprendizagem e de intervenção que considerem o efetivo papel dos trabalhadores. 
Nesse prisma, novamente, destacam-se os dois cursos de Medicina e o curso de Enfermagem do campus Arapiraca, ainda que os cursos de Odontologia e de Enfermagem do campus A. C. Simões também façam uma breve menção às metodologias ativas. Porém, são os três cursos já mencionados que desenvolvem o debate sobre essas metodologias com maior ênfase nos seus PPC, estruturando suas matrizes curriculares para privilegiá-las.

Assim, constatamos que a Saúde do Trabalhador, em conteúdo e forma, comparece no conjunto dos cursos de saúde da Ufal, direta ou indiretamente, mas ainda sem a amplitude que lhe cabe, se levado em conta que deveria ser um campo estruturante do SUS (Souza, 2019). Essa falta de amplitude reflete o caráter isolado e especializado que a Saúde do Trabalhador assumiu no SUS, mesmo com a proposta dos Cerest, incialmente, sendo a de difundir esse campo por todos os níveis de complexidade do sistema. No entanto, essa perspectiva não é consensual, pois alguns autores e profissionais da área (Costa et al., 2013) defendem que ela assuma, mesmo, o caráter de serviço especializado, pois demanda um tipo de conhecimento mais complexo que pode ser negligenciado em nível generalista.

A nosso ver, esse entendimento está em determinação recíproca com a parca inserção da Saúde do Trabalhador no ensino em saúde, a exemplo do que ocorre na Ufal, figurando mais em nível de Pós-Graduação (especialização) do que na graduação. Entra-se em uma espiral que resulta, cada vez mais, em uma Saúde do Trabalhador que se pretende (equivocadamente) atenção especializada e abandona seu papel, em tese, estrutural, transversal e central no sistema de saúde. Acreditamos que é preciso reverter essa lógica, conquanto qualquer serviço ou ação de saúde deve tomar a categoria trabalho como seu determinante elementar e, portanto, partir do princípio de que indivíduos e coletividades possuem seu processo saúde-doença consignados, sobremodo, ao trabalho. Uma formação em saúde que dê maiores espaços para a Saúde do Trabalhador pode contribuir para esse processo transformador.

\section{Considerações Finais}

Constatamos que a Saúde do Trabalhador é abordada, diretamente, por apenas três dos setes cursos de saúde da Ufal. Os dois cursos de Medicina e o curso de Enfermagem do campus Arapiraca apresentam componentes curriculares e proposta pedagógica com alguma convergência aos princípios da Saúde do Trabalhador. Os demais cursos apresentam componente nos quais há possibilidades para a abordagem das temáticas desse campo, a depender dos direcionamentos dados por seus docentes e as contingências da oferta efetiva do conteúdo. Em todo caso, o campo ainda deve ampliar sua inserção em todas as graduações, a fim de refletir seu papel estruturante ante o processo saúde-doença e o sistema de saúde.

Cabe frisar que esse estudo possui alguns limites, pois a análise dos PPC não reflete, em absoluto, o cotidiano de efetivação dos cursos, assim como deixa escapar as especificidades de cada oferta curricular, em face dos planos de disciplina e de aula. A percepção dos estudantes também deve ser objeto de investigação no bojo do problema abordado nesse artigo. Além disso, a presença da Saúde do Trabalhador em atividades de pesquisa e extensão deve constituir um viés para análises futuras, a fim de refletir a real dimensão que a área em questão possui no âmbito da Ufal, em toda sua complexidade. Aqui, apenas apresentamos uma análise preliminar que assume o status de ponto de partida para entender como se dá o ensino da Saúde do Trabalhador em Alagoas e, especificamente, na Ufal.

\section{Referências}

Costa, D., Lacaz, F. A. C., Jackson Filho, J. M. \& Vilela, R. A. G. (2013). Saúde do Trabalhador no SUS: desafios para uma política pública. Revista Brasileira Saúde Ocupacional. 38(127): 11-21.

Dias, E. C., Silveira, A. M., Chiavegatto, C. V. \& Resende, N. P. (2006). O ensino das relações trabalho-saúde-doença na escola médica: percepção dos alunos e proposta de aperfeiçoamento na UFMG. Revista Brasileira de Educação Médica. 30(1): 20-26.

Fernandes, M. C., Souza, N. V. D. O. \& Marques, C. F. (2013). Ensino da saúde do trabalhador na graduação de enfermagem: uma pesquisa bibliográfica. Revista Enfermagem UERJ. 21(supl 1): 254-260. 
Fernandes, M. C., Souza, N. V. D. O, Mafia I. F. \& David H. M. S. L. (2013). Potencialidades e limitações para o ensino da saúde do trabalhador em um curso de graduação em enfermagem. In: Anais do Seminário Nacional de Pesquisa em Enfermagem. Natal: Associação Brasileira de Enfermagem. 17: 312-314. http://www.abeneventos.com.br/anais_senpe/17senpe/pdf/0148co.pdf

Leão, L. H. C. \& Vasconcellos, L. C. F. (2011). Rede Nacional de Atenção Integral à Saúde do Trabalhador (Renast): uma rede que não enreda. In: Vasconcellos, L. C. F. \& Oliveira, M. H. B. O. (Orgs.). Saúde, trabalho, direito: uma trajetória crítica e a crítica de uma trajetória. Educam, 453-490.

Marques, C.F., Santos, D. M., Gonçalves, F. R., Fernandes, M. C. \& Souza N. V. D. O. (2012). O ensino de graduação e os conteúdos teórico-práticos da saúde do trabalhador. Revista Eletrônica de Enfermagem. 14(3): 494-503.

Mendes, R. \& Dias, E. C. (1991). Da medicina do trabalho à saúde do trabalhador. Revista de Saúde Pública. 25: 341-349.

Minayo-Gomez, C. \& Thedim-Costa, S. M. F. (1997). A construção do campo da saúde do trabalhador: percurso e dilemas. Caderno de Saúde Pública. 13(supl. 2): 21-32.

Monteiro, M. S., Santos, E. V., Kawakami, L. S. \& Wada M. (2007). O ensino de vigilância à saúde do trabalhador no Curso de Enfermagem. Revista da Escola de Enfermagem da USP. 41(2): 306-310.

Pereira Jorge I. M., Simonelli, A. P., Rosa, J. E., Ferreira, S. S. M., Souza, M. B. C. A. \& Barroso, B. I. L. (2016). Ensino de saúde do trabalhador nos cursos de graduação em Terapia Ocupacional: contribuições ao debate sobre parâmetros de avaliação do ensino público brasileiro. Revista de Terapia Ocupacional da Universidade de São Paulo. 27(2): 109-115.

Perin, P. C. P., Sales-Peres, S. H. C., Sales-Peres, A., Sales-Peres, A. C. \& Perin, L. F. M. G. (2012). A importância do ensino da odontologia do trabalho na graduação de odontologia. Revista da Faculdade de Odontologia de Lins/Unimep. 22(1): 39-45.

Souza, D. O. (2019). Saúde do(s) trabalhador(es): análise ontológica da "questão" e do "campo". Edufal.

Universidade Federal de Alagoas. (2007a). Curso de graduação em Enfermagem. Ufal.

Universidade Federal de Alagoas. (2007b). Projeto Pedagógico do curso de Odontologia da Ufal. Ufal.

Universidade Federal de Alagoas. (2007c). Projeto Político Pedagógico - curso de Farmácia. Ufal.

Universidade Federal de Alagoas. (2010). Projeto Pedagógico bacharelado em Nutrição. Ufal.

Universidade Federal de Alagoas. (2013). Projeto Pedagógico do curso de Medicina. Ufal.

Universidade Federal de Alagoas. (2018a). Projeto Pedagógico do curso de bacharelado em Enfermagem. Ufal.

Universidade Federal de Alagoas. (2018b). Projeto Pedagógico do curso de Medicina. Ufal.

Vasconcellos, L. C. F. (2011). Entre a saúde ocupacional e a saúde do trabalhador: as coisas nos seus lugares. In: Vasconcellos, L. C. F \& Oliveira, M. H. B. O. (Orgs.). Saúde, trabalho, direito: uma trajetória crítica e a crítica de uma trajetória. Educam. 401-422.

Vasconcellos, L. C. F., Almeida, C. V. B. \& Guedes, D. T. (2009). Vigilância em saúde do trabalhador: passos para uma pedagogia. Trabalho, Educação e Saúde. 7(3): 445-462. 